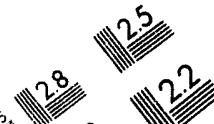

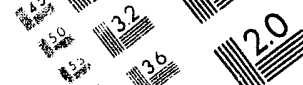
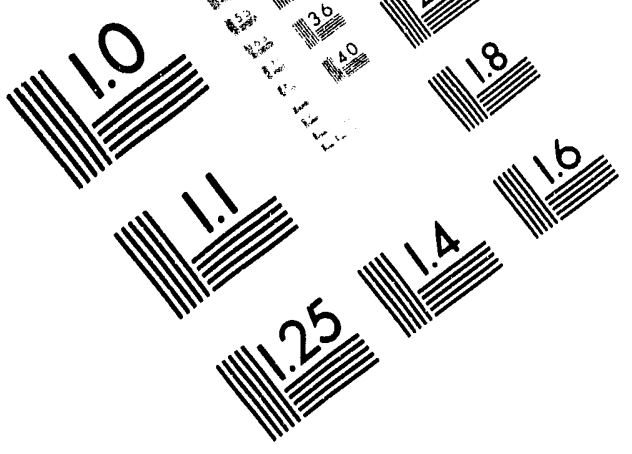

Association for Information and Image Management

1100 Wayne Avenue, Suite 1100

Silver Spring, Maryland 20910

301/587-8202

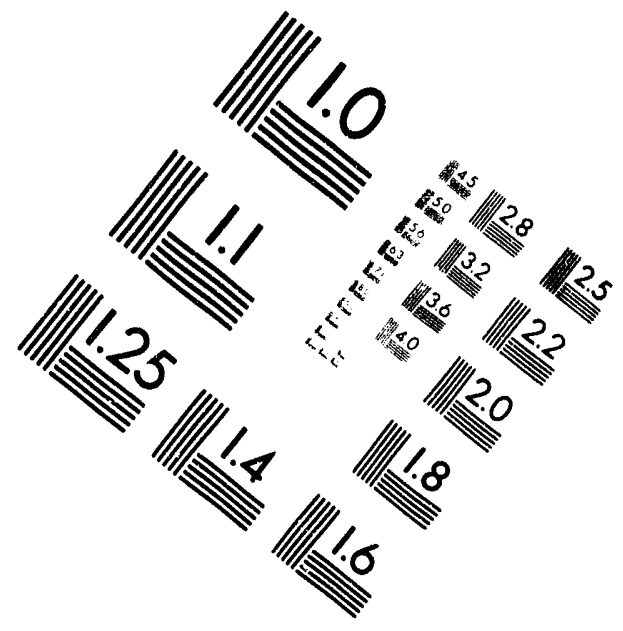

\title{
Centimeter
}

${ }_{1}$ Inches
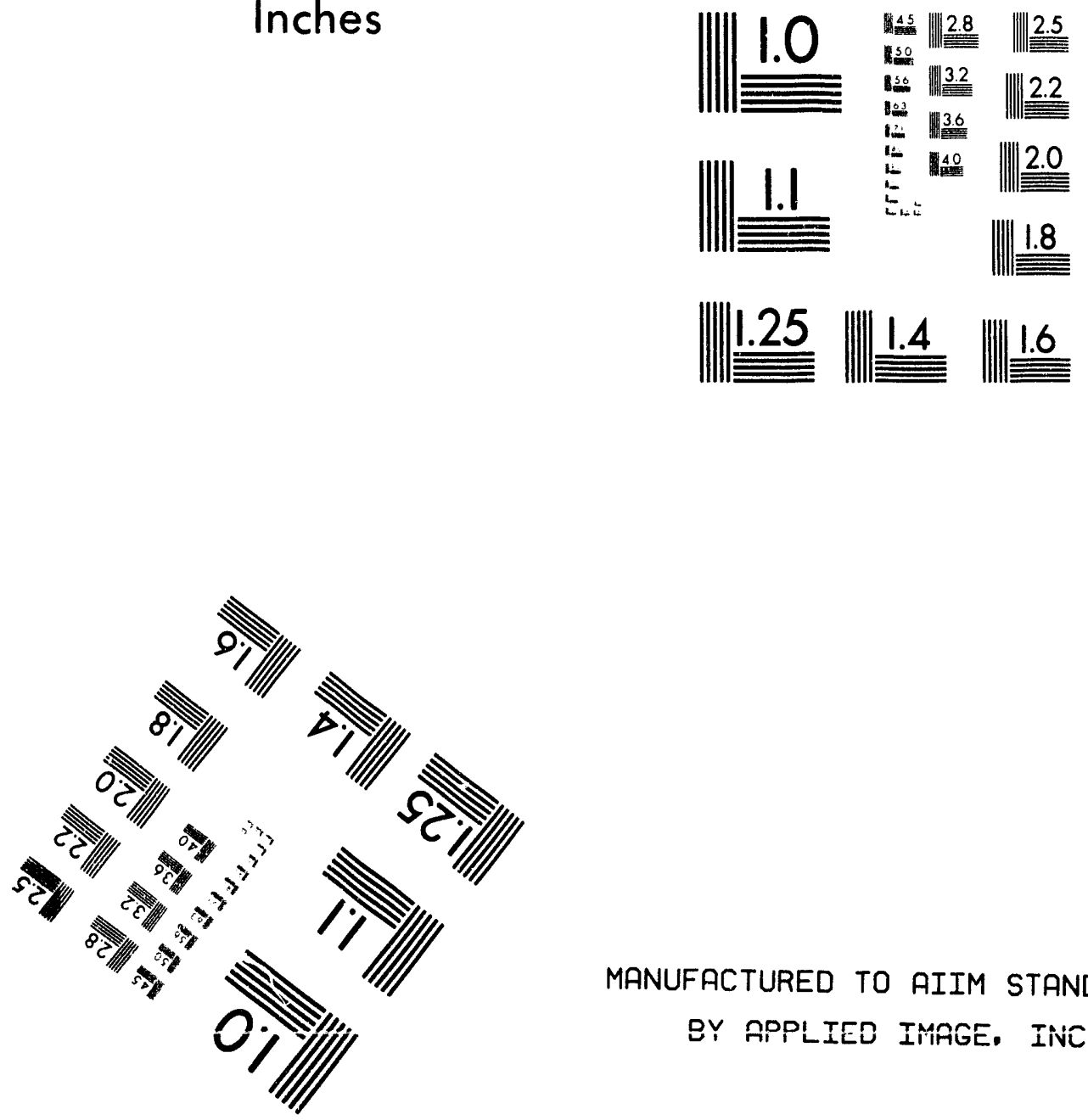

MANUFACTURED TO AIIM STANDARDS

QY APPLIED IMAGGE. INU.

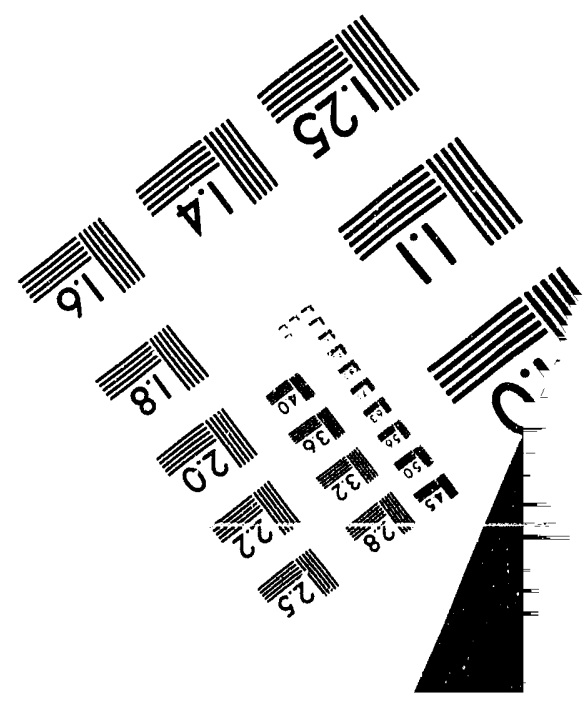



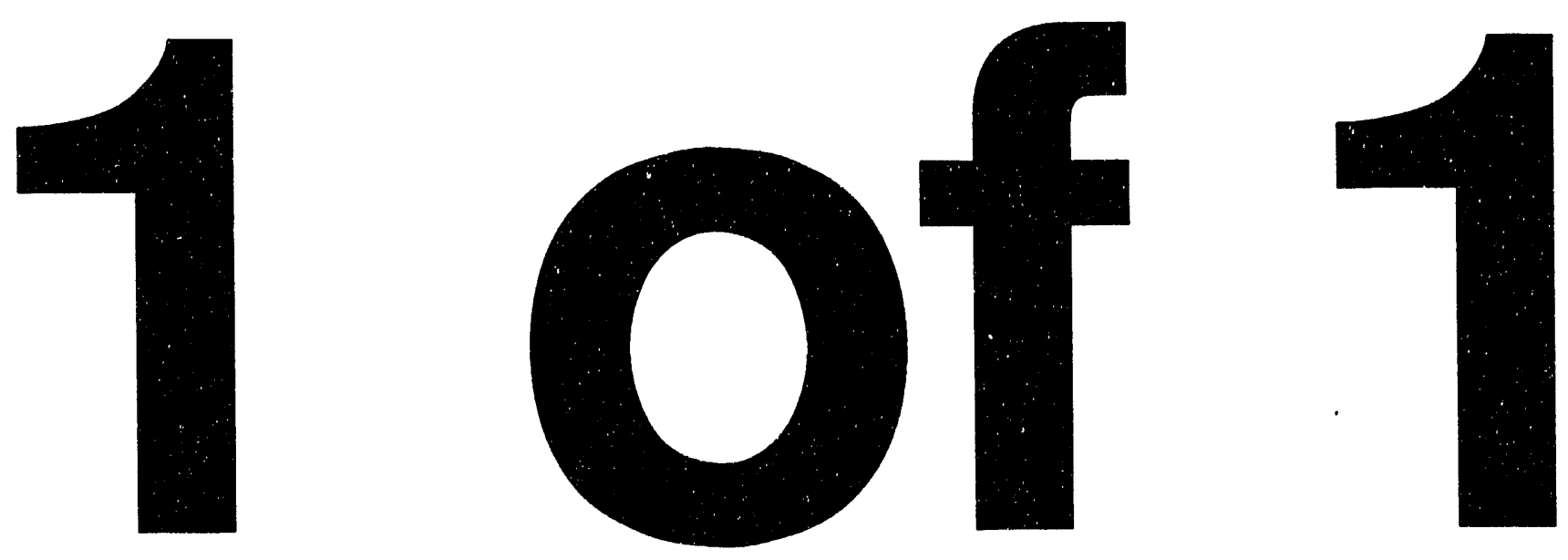


\section{Tracking Studies of Insertion Device Effects on Dynamic Aperture in the APS Storage Ring*}

Yong-chul Chae, E. A. Crosbie
Argonne National Laboratory, 9700 So. Cass Ave., Argonne, oflk 261993

\section{Abstract}

We studied the effects of an insertion device (ID) on the dynamic aperture in the 7-GeV Advanced Photon Source (APS) storage ring using the program RACETRACK [1]. We found that the nonlinear effect of the ID is the dominant effect on the dynamic aperture reduction compared to the other multipole errors which exist in the otherwise ideal lattice. The previous study of dynamic aperture [2] was based on the assumption that the effect of the fast oscillating terms in L. Smith's Hamiltonian [3] is small, and hence can be neglected in the simulation. The remarkable agreement between the previous study and the current results using RACETRACK, including all effects of the fast oscillating terms, justified those assumptions at least for the APS ring.

\section{INTRODUCTION}

The Advanced Photon Source (APS) storage ring is a 7-GeV third generation light source with forty straight sections. Intense $\mathrm{x}$-ray beams will be delivered by thirty-four undulators and wigglers. Thus the effects, linear and nonlinear, of the insertion devices on the beam dynamics becomes important. In this report we present the numerical tracking studies of the effect of IDs on the dynamic aperture.

\section{Planar Insertion Devices}

\section{A. Motivation for Using RACETRACK}

The field components for a planar insertion device (ID) suggested by $K$. Halbach [4] are given by:

$$
\begin{aligned}
B_{y} & =B_{o} \cosh k_{x} x \cosh k_{y} y \operatorname{cosk} z \\
B_{x} & =\frac{k_{x}}{k_{y}} B_{o} \sinh k_{x} x \sinh k_{y} y \cosh z \\
B_{z} & =-\frac{k}{k_{y}} B_{o} \cosh k_{x} x \sinh k_{y} y \operatorname{sink} z
\end{aligned}
$$

where

$$
k_{x}^{2}+k_{y}^{2}=k^{2}=\left(\frac{2 \pi}{\lambda_{w}}\right)^{2}
$$

and $\lambda_{w}$ is the period length.

The Hamiltonian with respect to the oscillating equilibrium orbit suggested by $L$. Smith [3] is given by:

$$
H=\frac{1}{2}\left(p_{x}^{2}+p_{y}^{2}\right)+
$$

*Work supported by U.S. Department of Energy, Office of Basic Energy Sciences under Contract No. W'-31-109-ENG-38.

iOn leave from University of Houston.

$$
\begin{aligned}
& \frac{1}{4 k^{2} \rho^{2}}\left[\cosh ^{2} k_{x} x \cosh ^{2} k \Omega+\frac{b_{x}^{2}}{\phi_{y}^{2}} s p h h^{2} k_{x} x \sinh ^{2} k_{y} y\right] \\
& -\frac{\sin k s}{k \rho}\left[p_{x}\left(\cosh k_{x} x \cosh k_{y} y-1\right)\right. \\
& \left.-\frac{k_{x}}{k_{y}} p_{y} \sinh k_{x} \sinh k_{y} y\right]
\end{aligned}
$$

Expanding to fourth order in $x$ and $y$, and assuming $k_{x}=0$, i.e. an ID with infinite width in the horizontal direction, Eq. 2 becomes

$$
H=\frac{1}{2}\left(p_{x}^{2}+p_{y}^{2}\right)+\frac{1}{2}\left(\frac{1}{2 \rho^{2}}-\frac{p_{x} k}{\rho} \sin k s\right) y^{2}+\frac{k^{2}}{12 \rho^{2}} y^{4},
$$

where $\rho$ is the radius of curvature in the peak field in the ID, $B_{0}$. We note that this Hamiltonian has a constant linear focusing term and an octupole-like term in the vertical direction if we neglect the fast oscillating (compared to betatron oscillation) term contained in $\sin (k s)$ for the moment. Neglecting the $\sin (k s)$ term, we may treat the ID as a long quadrupole with a lumped nonlinear element at the center. The equations of motion may be written as

$$
\frac{d^{2} y}{d s^{2}}=\frac{-\sinh (2 k y)}{4 k \rho^{2}}, \quad \frac{d^{2} x}{d s^{2}}=0,
$$

where $s$ is the equilibrium orbit length. The previous tracking study based on this approach was done using the PACMAN code [2]. We found that the dynamic aperture was larger than the physical aperture at the ID section.

As a continuation of this effort, including the effects of fast oscillating terms, we decided to use RACETRACK for dynamic aperture study. The program, developed at Sincrotrone Trieste, has a unique abilility to perform secondorder symplectic integration for motion in the insertion device $[5,6]$.

\section{B. Effects of an ID on Dynamic Aperture}

One of the major insertion devices in the APS project is the Type-A undulator with peak field $B_{0}=0.85 \mathrm{~T}$, undulator period $\lambda_{w}=3.3 \mathrm{~cm}$, total ID length $L_{I D}=2.31 \mathrm{~m}$. The constant linear vertical focusing strength for this ID is

$$
\frac{L_{I D}}{2 \rho^{2}}=0.0015\left(m^{-1}\right)<<K_{Q}=0.51147\left(m^{-1}\right)
$$

where $K_{Q}$ is a typical quadrupole strength in the APS ring. Hence the linear effect of the ID considered here will be very small. In order to investigate the effect of oscillating terms, we first note that $p_{x}$ is constant for each pass of the particle through the ID. The equation of motion is therefore a Mathiew equation. Examination of this equation shows that the forucings effert of t!̣e oscil!ating 


\section{DISCLAIMER}

This report was prepared as an account of work sponsored by an agency of the United States Government. Neither the United States Government nor any agency thereof, nor any of their employees, makes any warranty, express or implied, or assumes any legal liability or responsibility for the accuracy, completeness, or usefulness of any information, apparatus, product, or process disclosed, or represents that its use would not infringe privately owned rights. Reference herein to any specific commercial product, process, or service by trade name, trademark, manufacturer, or otherwise does not necessarily constitute or imply its endorsement, recommendation, or favoring by the United States Government or any agency thereof. The views and opinions of authors expressed herein do not necessarily state or reflect those of the United States Government or any agency thereof. 
term was negligible for acceptable va!. ; of $p_{r}$. 'This is verified by comparing the linear tune shifts obtained from the analytic estimation and the numerical result of $\mathrm{RACE}-$ TRACK. Thus, the linear effect of the II) on the dynamic aperture reduction is very small. This is clearly scen in Fig. 1 which shows that dynamic aperture reduction is mainly due to the nonlinear effect of the ID

Since the lattice elements in the ring have error ficlds due to imperfect construction, the effects of the multipole errors in the lattice were investigated. For this purpose, the random normal sextupole and octupole field errors based on the APS magnetic field tolerance budget were included in the dipole and quadrupole magnets. The rms values of these multipole errors are $b_{2 D}=5 \times 10^{-5}, b_{3 D}=1 \times 10^{-5}$, $b_{2 Q}=1 \times 10^{-4}$ and $b_{3 Q}=5 \times 10^{-5}$ where $b_{n . D}$ and $b_{n Q}$ are the normal $2(n+1)$-pole field coefficients in the dipole and quadrupole magnets, respectively. Field error coefficients are defined as

$$
B_{y}+i B_{x}=B_{0} \sum_{n=0}\left(b_{n}+i a_{n}\right)(x+i y)^{n},
$$

where $b_{n}$ and $a_{n}$ indicate the normal and skew components, respectively. Although these error fields reduce the dynamic aperture of an ideal lattice without an ID, we expected that, because ID effects on dynamic aperture reduction are so dominant, the inclusion of the multipole errors in the ordinary lattice would not cause much additional change to a dynamic aperture already reduced by the presence of the ID. This is verified in Fig. 2.

The results for multiple IDs are also shown in Fig. 1. As expected, the dynamic aperture is further reduced as the number of IDs around the ring increases. However, the aperture reduction for 20 IDs is no worse than that for 10 IDs. The dynamic aperture is still larger than the physical aperture.

Although not shown here, we investigated the effect of the non-zero horizontal wave number $\left(k_{x}\right)$. Depending on whether $k_{x}$ is real or imaginary, such an ID will provide additional horizontal focusing and defocusing, respectively. We found the tracking results were essentially the same in the realistic variation of $k_{x}$ values.

Finally, we want to mention that when we compare the tracking results from the programs PACMAN and RACETRACK the phase space as well as dyanamic aperture show remarkable agreement (see Fig. 3 for the comparison of dynamic aperture). Thus, we may tentatively conclude that the effect of the fast oscillating terms which appeared in L. Smith's IIamiltonian will be small for the IDs in the APS storage ring.

\section{Helical Insertion Devices}

There is an increasing interest in the utilization of circularly or elliptically polarized light for some types of experiments. Such a light will be delivered by helical IDs. One type of helical ID) considered in the APS project is the crossed insertion device originally conceptualized by
Onuki [7]. The magnetic fields of crossed IDs are the superposition of the horizontal and vertical planar IDs whose field expressions, in simplified form, may be written as

$$
B_{x}=B_{0}^{\prime} \sin (k z), B_{y}=B_{0} \sin (k z-\alpha), B_{z}=0,
$$

where $B_{0}^{\prime}$ and $B_{0}$ are the peak ficld in the $\mathrm{x}$ - and $\mathrm{y}$ directions, respectively, $k$ is the usual wave number, and $\alpha$ is the longitudinal phase slip between the horizontal and vertical planar IDs. By adjusting $\alpha$, the field projected onto the $x-y$ plane will be either circular or elliptic. For instance, if $B_{0}$ is equal to $B_{0}$, the field will look circular if $\alpha=\frac{\pi}{2}$ or $\frac{3 \pi}{2}$ and elliptic if $\alpha=\frac{3 \pi}{4}$ or $\frac{7 \pi}{4}$.

In the APS, such a helical ID has the parameters $B_{0}=$ $1 T, B_{0}^{\prime}=0.07 T, \lambda_{\omega}=15 \mathrm{~cm}$, and total length of device $L_{I D}=3 \mathrm{~m}$. Since the deflection parameter $K$, defined by

$$
K=0.934 \lambda_{w}(\mathrm{~cm}) B_{0}(T),
$$

is equal to 14 for this device, it has the focusing characteristics of a wiggler. In general, the dominant effect of a wiggler is conceived as linear because it usually requires a higher peak field and a longer period length than an undulator. Such a linear effect results in dynamic aperture reduction through breaking the symmetry of the lattice. Thus, a sophitiscated matching scheme may be required to restore the linear optics. However, for the APS helical ID, the distortion of linear optics is found to be very small mainly because the beam energy is high and the peak field is relatively low comparable to the planar Type-A ID considered in the previous section.

Also, the nonlinear effect of the helical ID will be small compared to the effect of the Type-A ID since the helical ID has a longer period length than the Type-A ID. We may expect that the dynamic aperture reduction due to the single helical ID should not be worse than that for the single Type-A ID. This is seen in Fig. 4 which shows the tracking results from RACE'TRACK for the various values of the $B_{0}^{\prime}$ field.

\section{CONClusion AND Discussion}

A study of the effects of an insertion device on the dynamic aperture is presented in this report. We found that, for Type-A and helical insertion devices considered for the APS project, the nonlinear effect was the dominant cause of dynamic aperture reduction. Also, with up to 20 IDs in the ring, stable amplitude of the beam oscillation is still outside of the physical vacuum chamber.

We also found that when we compared the numerical results from RACETRACK with the one from Ed Crosbie's PACMAN, the agreement between the two were remarkable. Hence, we may tentatively conclude that the effects of the fast oscillating terms contained in the ID Hamiltonian are negligible.

\section{ACKNOWLEDGEMENTS}

'The discussion with Dr. R. Nagaoka: who visited APS 
from Sincrotrone 'Trieste, was most helpful to us in this study.

\section{REFERENCES}

[1] F. lazmourene, et al., "RACETRACK USER'S GUIDE VERSION 4," Sincrotrone Trieste, July, 1991.

[2] "7-GeV Advanced Photon Source Conceptual Design Report," ANL-87-15, 1987.

[3] L. Smith, "Efrects of Wigglers and Undulators on Beam Dynamics," LBL ESG TECH NOTE-24, September, 1986.

[4] K. Ilalbach, "Physical and Optical Properties of Rare Earth Cobalt Magnets," NIM, Vol 187, 1981.

[5] R. Nagaoka and L. Tosi, "An Improved Schente for Integrating the Particle Motion through the Inscrtion Device in RACETRACK," ST/M-90/6, Sincrotrone Trieste, March, 1990.

[6] R. Nagaoka and L. Tosi, "An Extended Scheme of the New Tracking Routine for Nonplanar Insertion Devices," ST/M-91/4, Sincrotrone Trieste, May, 1991.

[7] H. Onuki, "Elliptically Polarized Synchrotron Radiation Source with Crossed and Retarded Magnetic Fields," NIM, Vol. 246, 1986.

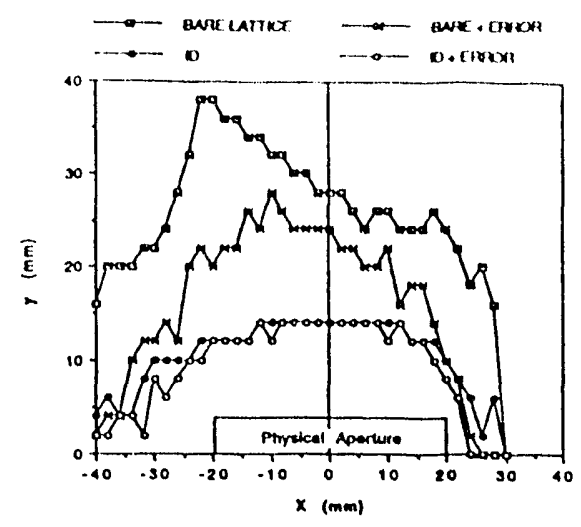

Figure 2

Dynamic aperture reduction due to a single ID with and without the multipole errors in the lattice.

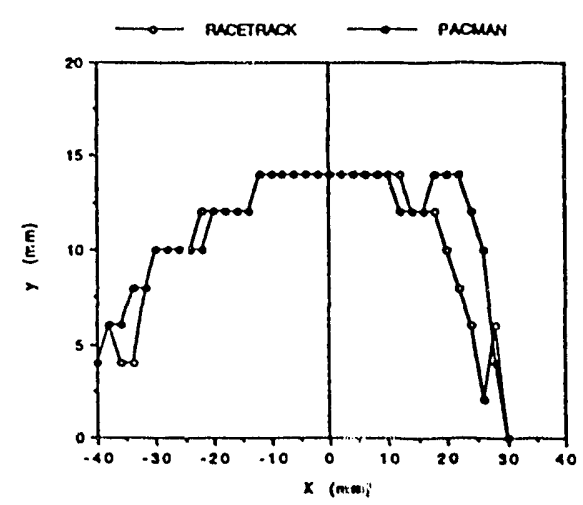

Figure 3

Dynamic aperture reduction due to a single ID (Comparison of results from the PACMAN and RACETRACK programs).

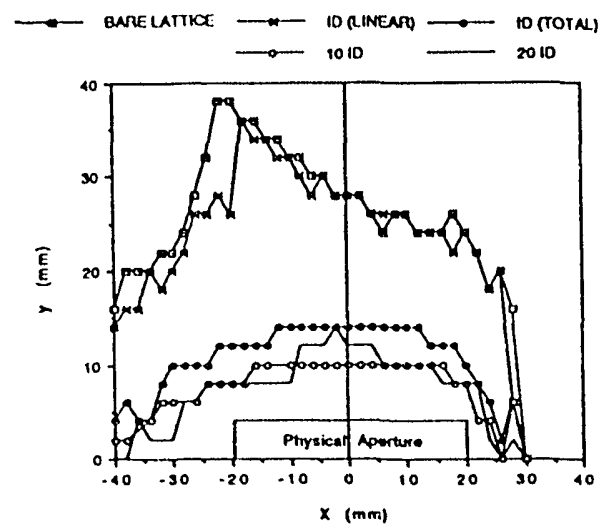

Figure 1

Dynamic aperture reduction due to a single ID) (linear effect vs. total effect) and due to multiple IDs.

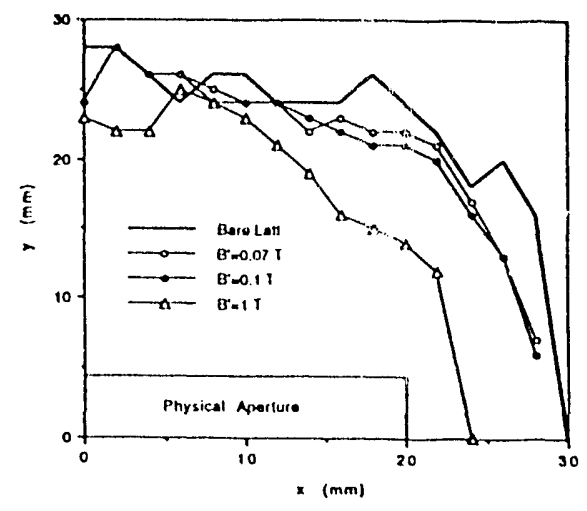

Figure 4

Dynamic aperture reduction due to a single helical ID $\left(B_{0}=1.0 \mathrm{~T}\right.$ and $\left.B_{0}^{\prime}=0.07 \mathrm{~T}, 0.1 \mathrm{~T}, 1.0 \mathrm{~T}\right)$. 

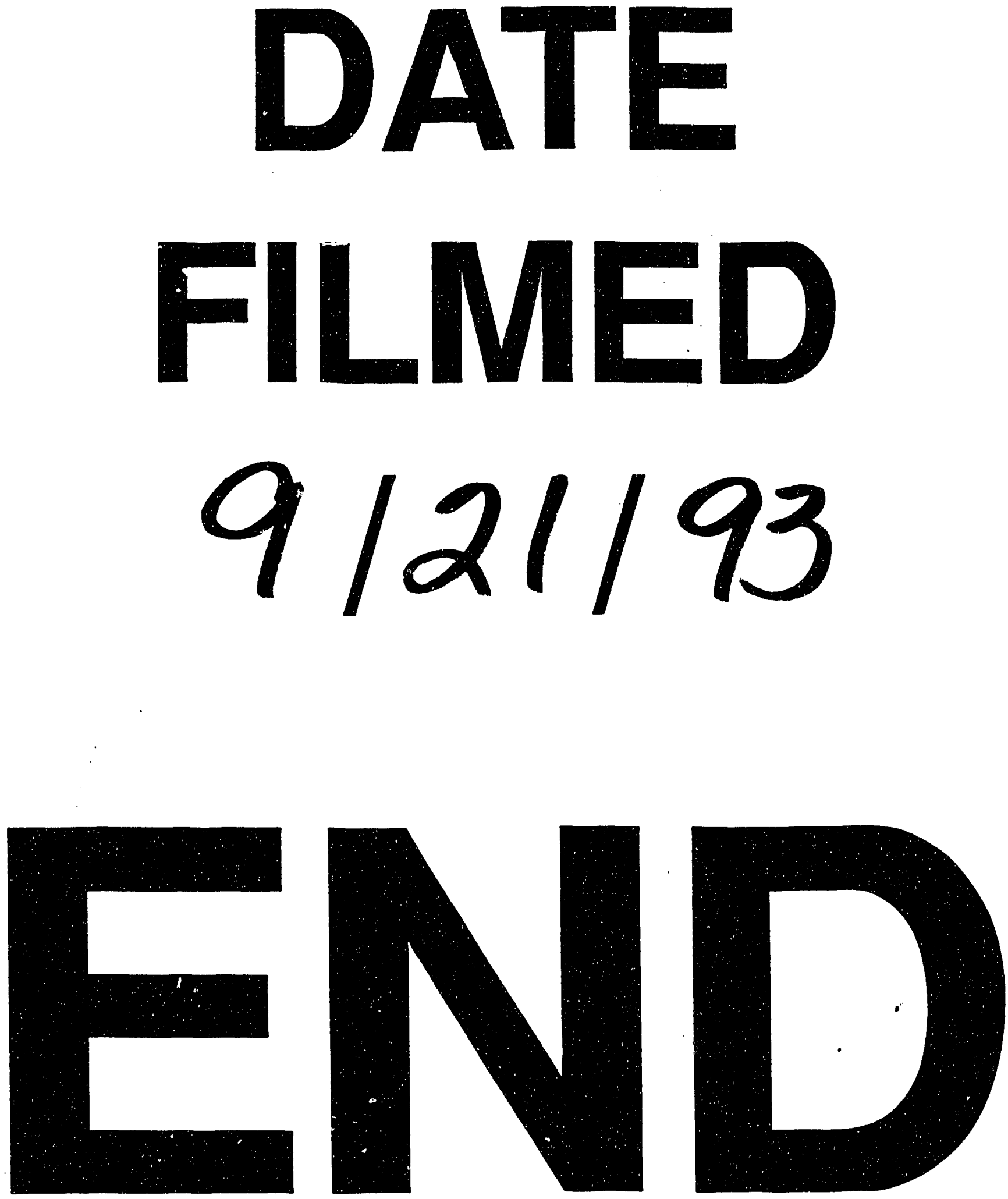
